\title{
ON RIGHT SELF-INJECTIVE REGULAR SEMIGROUPS, II
}

\author{
KUNITAKA SHOJI
}

(Received 2 November 1981)

Communicated by T. E. Hall

\begin{abstract}
It is shown that a semigroup is right self-injective and a band of groups if and only if it is isomorphic to the spined product of a self-injective semilattice of groups and a right self-injective band. A necessary and sufficient condition for a band to be right self-injective is given. It is shown that a left [right] self-injective semigroup has the [anti-] representation extension property and the right [left] congruence extension property.
\end{abstract}

1980 Mathematics subject classification (Amer. Math. Soc.): $20 \mathrm{M} 10$

\section{Introduction}

Let $S$ be a semigroup and let $M_{S}$ be a non-empty set $M$ with an operation of $S$ on the right side. Then $M_{S}$ is called a right $S$-system if $m(s t)=(m s) t$ for all $m \in M_{S}$ and for all $s, t \in S$. Let $\phi$ be a mapping of $A_{S}$ into $B_{S}$, where both $A_{S}$ and $B_{S}$ are right $S$-systems. Then $\phi$ is called an $S$-homomorphism if $\phi(a s)=\phi(a) s$ for all $a \in A_{S}$ and for all $s \in S$. A right $S$-system $M_{S}$ is called injective if for any injective $S$-homomorphism $\psi: A_{S} \rightarrow B_{S}$ and for any $S$-homomorphism $\xi: A_{S} \rightarrow M_{S}$, there is an $S$-homomorphism $\delta: B_{S} \rightarrow M_{S}$ such that $\delta \psi=\xi$. A semigroup $S$ is called right self-injective if the right $S$-system $S_{S}$ is injective. Dually, a left self-injective semigroup is defined. A both left and right self-injective semigroup is called self-injective. Schein [7] has characterized self-injective inverse semigroups as follows.

Let $S$ be an inverse semigroup with semilattice of idempotents $E$. A subset $B$ of $S$ is compatible if $B^{*} B \subseteq E, B B^{*} \subseteq E$, where $B^{*}$ is the set of inverses of elements of $B$. Define an order $\leqslant$ on $S$ by $a \leqslant b(a, b \in S)$ if and only if $a \in b E$. Then (i)

C. Copyright Australian Mathematical Society 1983 
$S$ is called complete if every compatible subset $B$ of $S$ has a least upper bound $\vee B$ with respect to $\leqslant$, (ii) $S$ is called infinitely distributive if ( $\vee B) a=\vee(B a)$ for any compatible subset $B$ of $S$ and for any $a \in S$ and (iii) $S$ is called E-reflexive if $s t \in E(s, t \in S)$ implies $t s \in E$.

RESULT 1.1 [7, Theorem 2.3]. Let $S$ be an inverse semigroup with semilattice of idempotents $E$. Then $S$ is self-injective if and only if $S$ is complete, infinitely distributive and E-reflexive.

According to Venkatesan [11], a regular semigroup $S$ is called right [left] inverse if each principal left [right] ideal of $S$ contains a unique idempotent generator.

The author [10] showed that a right self-injective generalized inverse [right $H$-compatible regular, 0-proper regular] semigroup is right inverse and gave a structure theorem for right self-injective generalized inverse semigroups. In Section 2, we shall state some results on a right self-injective, right inverse semigroup. We shall prove a structure theorem for right self-injective bands of groups. In Section 3, we shall give characterisations of certain right self-injective bands (almost commutative bands and chains of finite right zero semigroups). In Section 4, we shall show that a left [right] self-injective semigroup has the [anti-] representation extension property and the right [left] congruence extension property in the sense of Hall [4]. Throughout this paper, we shall use the following notations.

For a semigroup $S, S^{1}\left[S^{0}\right]$ is the semigroup obtained from $S$ by adjoining an identity 1 [zero 0].

For a regular semigroup $S, E(S)$ is the set of all idempotents of $S$. For any $x \in S, x^{*}$ is an inverse of $x$ (that is, $x x^{*} x=x, x^{*} x x^{*}=x^{*}$ ) and $V(x)$ is the set of all inverses of $x$ in $S$. Further, $x^{-1}$ denotes the group-inverse of $x$ (that is, $x^{-1} \in V(x)$ and $\left.x x^{-1}=x^{-1} x\right)$ if it exists. For any subset $M$ of $S, M^{*}=\cup\{V(a) \mid$ $a \in M\}$. All other notations and terminology should be referred to [1] and [10]. unless otherwise stated.

\section{Right self-injective, right inverse semigroups}

We shall collect several known results on right inverse semigroups.

Result 2.1 ([2], [11], [13]). Let $S$ be a right inverse semigroup.

(i) For any $f, h \in E(S), h f h=f h$, that is, $E(S)$ is a right regular band in the sense of Kimura [6].

(ii) The intersection of two principal right ideals of $S$ is a principal right ideal of $S$. 
(iii) For any $a, b \in S, b^{*} a^{*} \in V(a b)$.

(iv) For any $a \in S, a^{*} a=a^{\prime} a$ for all $a^{*}, a^{\prime} \in V(a)$.

(v) The relation $\sigma=\{(a, b) \in S \times S \mid V(a)=V(b)\}$ is the finest inverse semigroup congruence on $S$.

(vi) For any $a \in S, a \sigma \in E(S / \sigma)$ and only if $a \in E(S)$. In this case, the subset ao of $S$ forms a right zero semigroup.

(vii) For any $a, b \in S, a=b$ if and only if $a \sigma b$ and $a^{*} a=b^{*} b$.

RESULT 2.2 [10]. Let $S$ be a right self-injective, right inverse semigroup.

(i) $S$ has a left identity element and a zero element.

(ii) Each principal right ideal of $S$ is injective.

(iii) For each right ideal $K$ of $S$, there is a unique principal right ideal $K^{\prime}$ of $S$ such that $K^{\prime} \supseteq K$ and for any distinct $x, y \in K^{\prime}$, there is an element $s \in S^{1}$ satisfying $x s \neq y s, x s, y s \in K$. In this case, $K$ is said to be strictly large in $K^{\prime}$.

(iv) The family of all principal right ideals of $S$ is a complete, infinitely distributive lattice under set-inclusion.

COROLlary 2.3. Let $S$ be as in Result 2.2. Then

(i) $E(S)$ is a right regular band with zero and left identity elements

(ii) for each right ideal $J$ of $E(S)$, there is a principal right ideal $J^{\prime}$ in which $J$ is strictly large.

Proof. (i) This follows from Result 2.1(i) and Result 2.2(i).

(ii) Let $J$ be any right ideal of $E(S)$. Then $J S$ is a right ideal of $S$. By Result 2.2(iii), there is an idempotent $e \in S$ such that $J S$ is strictly large in $e S$. Let $a, b \in e E(S)$ with $a \neq b$. Then $a s, b s \in J S$, as $\neq b s$ for some $s \in S^{1}$. Hence, $a\left(s s^{*}\right), b\left(s s^{*}\right) \in J, a\left(s s^{*}\right) \neq b\left(s s^{*}\right)\left(s^{*}=1\right.$ if $\left.s=1\right)$. Therefore, $J$ is strictly large in $e E(S)$, proving the corollary.

LEMMA 2.4. Let $S$ be a right self-injective, right inverse semigroup and let $M$ be a subset of $S$ such that $M M^{*}, M^{*} M \subseteq E(S)$. Then there is an element $w \in S$ such that

$$
\begin{gathered}
M S \text { is strictly large in } w S, \\
w a^{*} a=a \text { for all } a \in M \text { and } a^{*} \in V(a) .
\end{gathered}
$$

If there is any other element $z \in S$ satisfying (2.1), (2.2), then $z w^{*} w=w, w z{ }^{*} z=z$.

Proof. Let $a, b \in M$. Then for any $x \in a^{*} a S \cap b^{*} b S$, $a x=$ $a\left(b^{*} b\right)\left(a^{*} a\right)\left(b^{*} b\right) x=\left(a b^{*}\right)\left(b a^{*}\right)\left(a b^{*}\right) b x=\left(b a^{*}\right)\left(a b^{*}\right)(b x)$ (by Result 2.1(i)) = $b x$. Thus we can define an $S$-homomorphism $\phi: M^{*} S \rightarrow S$ by $\phi(t)=a t$ if 
$t \in a^{*} a S, a \in M$. Suppose, $x, y \in M^{*} S, \phi(x)=\phi(y)$. Then $x \in a^{*} a S, y \in b^{*} b S$ for some $a, b \in M$ and $a x=b y$. Hence, $x=\left(a^{*} a\right) x=a^{*} b y=\left(a^{*} b\right)\left(b^{*} b\right) y=$ $\left(b^{*} b\right)\left(a^{*} b\right)\left(b^{*} b\right) y$ (by Result 2.1(i)). Therefore, $x \in a^{*} a S \cap b^{*} b S$. Similarly, $y \in$ $a^{*} a S \cap b^{*} b S$. Then it follows from the above that $a x=b x, a y=b y$. Hence, $x=a^{*} a x=a^{*}(b y)=a^{*}(a y)=y$. This means that $\phi$ is injective. By Result 2.2(iii), there is an idempotent $f \in S$ such that $M^{*} S$ is strictly large in $f S$. Since $S$ is right self-injective, there is an $S$-homomorphism $\tilde{\phi}: f S \rightarrow S$ with $\tilde{\phi} \mid M^{*} S=\phi$. Since $M^{*} S$ is strictly large in $f S$, it follows that $\tilde{\phi}$ is injective. Put $w=\tilde{\phi}(f)$. Then it follows immediately that $w$ satisfies (2.1), (2.2). Suppose that there is some other element $z \in S$ satisfying (2.1), (2.2) and that $z w^{*} w \neq w$. Then by Result 2.2(iii), $z S=w S$. So there must be an element $s \in S^{1}$ such that $z w^{*} w s$, ws $\in M S$ but $z w^{*} w s \neq w s$. Then $w s=a t$ for some $a \in M$ and $t \in S$. Hence, $w^{*} w s=w^{*} a t=$ $w^{*}\left(w a^{*} a\right) t=\left(a^{*} a\right)\left(w^{*} w\right)\left(a^{*} a\right) t$ (by Result 2.1(i)) $=\left(w a^{*} a\right)^{*}\left(w a^{*} a\right) t$ (by Result $2.1\left(\right.$ iii), (v)) $=a^{*}$ at (by $\left.(2.2)\right)$. Thus, $z\left(w^{*} w s\right)=z\left(a^{*} a t\right)=a t$ (by $\left.(2.2)\right)=w s$, a contradiction. The lemma is proved.

LeMma 2.5. Let $S$ be as in Lemma 2.4. If $x y \in E(S)(x, y \in S)$ then $y x \in E(S)$.

Proof. Let $x^{*} \in V(x), y^{*} \in V(y)$. Define a mapping $\phi: x^{*} S \cup y S \rightarrow x S \cup y^{*} S$ by $\phi(a)=x a$ if $a \in x^{*} S$ and $\phi(a)=y^{*} a$ if $a \in y S$. Then for any $b \in x^{*} S \cap y S$, $x b=x\left(y y^{*}\right)\left(x^{*} x\right) b=\left(y^{*} x^{*}\right)(x y)\left(y^{*} x^{*}\right) x b$ (by Result 2.1(i) and the fact that $y^{*} x^{*} \in E(S)$, by Result 2.1(iii), (vi)) $=y^{*}\left(x^{*} x\right)\left(y y^{*}\right)\left(x^{*} x\right) b=y^{*} b$. Therefore, $\phi$ is well-defined. Since $S$ is right self-injective, there is an $S$-homomorphism $\tilde{\phi}$ : $S \rightarrow S$ with $\tilde{\phi} \mid\left(x^{*} S \cup y S\right)=\phi$. By virtue of Result $2.2(\mathrm{i})$, let $h$ be a left identity element of $S$. Put $z=\tilde{\phi}(h)$. Then $z x^{*} x=x$ and $z y y^{*}=y^{*}$. Thus, $y z=\left(y y^{*} y\right) z$ $=y\left(z y y^{*}\right) y z=y z y z$, that is, $y z \in E(S)$. Hence, $y x=y\left(z x^{*} x\right)=y z\left(x^{*} x\right) \in E(S)$. The lemma is proved.

THEOREM 2.6. Let $S$ be a right self-injective, right inverse semigroup. Then $S / \sigma$ is a self-injective inverse semigroup.

Proof. By Result 2.1(v), $S / \sigma$ is an inverse semigroup. By Result 1.1, it suffices to show that $S / \sigma$ is complete, infinitely distributive and $E$-reflexive. Let $H$ be any compatible subset of $S / \sigma$. If $H$ is an empty set, then a zero element of $S / \sigma$ is the least upper bound of $H$ (note that $S / \sigma$ has a zero element, since $S$ has a zero element, by Result 2.2(i)). So we assume that $H$ is a non-empty set. Put $M=\{x \in S \mid x \sigma \in H\}$. Then it follows from Result 2.1(vi) that $M M^{*}, M^{*} M \subseteq$ $E(S)$. By Lemma 2.4, there is an element $w \in S$ satisfying (2.1), (2.2). It will be seen that $w \sigma$ is the least upper bound of $H$. Let $z$ be any element of $S$ such that $z \sigma$ is an upper bound of $H$. Then $z a^{*} a \sigma a$ for all $a \in M$ and $a^{*} \in V(a)$. In this case, 
we have $z a^{*} \sigma a a^{*}$. So by Result 2.1(vi), we get $z a^{*} \in E(S), z a^{*} \Re a a^{*}$. Hence, $a=z a^{*} a$ for all $a \in M$ and $a^{*} \in V(a)$. Then it is easily checked that $w w^{*} z$ satisfies (2.1), (2.2). By Lemma 2.4, we have $\left(w w^{*} z\right) w^{*} w=w$. Hence, $\left(z w^{*}\right)\left(z w^{*}\right)$ $=z\left(w^{*} w^{*}\right) z\left(w^{*} w w^{*}\right)=\left(z w^{*}\right) w w^{*}=z w^{*} \in E(S)$. Thus, $z w^{*} w=z\left(w^{*} w w^{*}\right) w$ $=\left(w w^{*}\right)\left(z w^{*}\right)\left(w w^{*}\right) w($ since $E(S)$ is right regular, by Result $2.1(\mathrm{i}))=w\left(w^{*} w\right)=$ $w$, whence, $z \sigma \geqslant w \sigma$. Therefore, $w \sigma$ is the least upper bound of $H$, and $S / \sigma$ is complete. Also it follows from Result (2.1)(vi) and Lemma 2.5 that $S / \sigma$ is $E$-reflexive. Let $P_{r}(S)$ denote the family of all principal right ideals of $S$. By using Result 2.1(vi), we can find a one-to-one correspondence between $P_{r}(S)$ and $E(S / \sigma)$. This gives an isomorphism of $P_{r}(S)$ onto $E(S / \sigma)$ as semilattices. So by Result 2.2(iv), $E(S / \sigma)$ is infinitely distributive. As noted in [7, page 531], we obtain that $S / \sigma$ is infinitely distributive. The proof of the theorem is complete.

LEMMA 2.7. Let $S$ be a right inverse semigroup which is a union of groups.

(i) $S$ is a semilattice $\Lambda$ of right groups $S_{\alpha}(\alpha \in \Lambda)$, each $S_{\alpha}$ being an $\Re$-class.

(ii) Each right ideal of $S$ is an ideal of $S$.

(iii) $S / \sigma$ is a semilattice of groups.

Proof. (i) This follows from Theorem 4.6 of [1] and Result 2.1(i).

(ii) By (i), we can obtain that $a b \Re b a$ for all $a, b \in S$. Thus we get $S a \subseteq a S$ for all $a \in S$. This implies (ii).

(iii) By Result 2.1(v), $S / \sigma$ is an inverse semigroup, while, clearly, $S / \sigma$ is a union of groups. It follows from Theorem 4.6 of [1] that $S / \sigma$ is a semilattice of groups, proving the lemma.

LeMMA 2.8. Let $S$ be as in Lemma 2.7. Let $J, K$ be right ideals of $S$ and let $\psi$ be an $S$-homomorphism of $K \rho^{\natural}$ onto $J$, where $\rho$ is a right congruence on $S$.

(i) $K \supseteq J$.

(ii) For any $a \in J, a \Re \psi(a \rho)$.

(iii) For any $a, b \in J, \psi(a \rho)=\psi(b \rho)$ implies $a=b$.

Proof. (i) Let $a \in J$. Then $a=\psi(k \rho)$ for some $k \in K$. So $a k^{-1} k=a$. By Lemma 2.7(ii), $k S$ is an ideal of $S$. Hence, $a \in k S$. Thus we get $J \supseteq K$.

(ii) Let $a \in J$. Then $a=\psi(k \rho)$ for some $k \in K$. So, $a=\psi\left(\left(k a^{-1} a\right) \rho\right)=$ $\psi\left(\left(k k^{-1} k a^{-1} a\right) \rho\right)=\psi\left(\left(k k^{-1}\right) \rho\right) k a^{-1} a$. By Lemma 2.7(i), $a \in k a^{-1} a S \subseteq a S$. This implies that $a \Re k a^{-1} a$. From Lemma 2.7(ii), we have $a, k a^{-1} a$ are contained in a right group $R$. Thus $a=\left(k a^{-1} a\right) t$ for some $t \in R$. Then $\psi(a \rho)=\psi\left(\left(k a^{-1} a t\right) \rho\right)=$ $a t$, and then $\psi(a \rho), a \in R$. Therefore, $a \mathscr{R} \psi(a \rho)$.

(iii) Let $a, b \in J$ with $\psi(a \rho)=\psi(b \rho)$. Then by (ii), $a \Re \psi(a \rho)=\psi(b \rho) \Re b$. Also from Lemma 2.7(i), $a, b, a a^{-1}, \psi\left(\left(a a^{-1}\right) \rho\right)$ are contained in a right group. So 
we get $a=b$, since $\psi\left(\left(a a^{-1}\right) \rho\right) a=\psi(a \rho)=\psi(b \rho)=\psi\left(\left(a a^{-1}\right) \rho\right) b$. The lemma is proved.

THEOREM 2.9. Let $S$ be a right inverse semigroup which is a union of groups. Then $S$ is right self-injective if and only if $S$ satisfies the following conditions (i) to (iv):

(i) $S$ has a zero element;

(ii) $S / o$ is a complete inverse semigroup;

(iii) for each right ideal $I$ of $S$, there is a principal right ideal $I^{\prime}$ of $S$ in which $I$ is strictly large;

(iv) for any principal right ideal $H$ of $S$ and for any right congruence $\rho$ on $S$ with $\rho \cap(H \times H)=\iota_{H}$, there is an idempotent $h \in S$ such that $h S=H$ and $a \rho b$ $(a, b \in S)$ implies $h a=h b$.

Proof. Suppose $S$ is right self-injective. Condition (i) follows from Result 2.2(i), condition (ii) follows from the proof of Theorem 2.6, and condition (iii) follows from Result 2.2(iii). Let $H, \rho$ be as in condition (iv). Then there is an $S$-homomorphism $\psi: H \rho^{\natural} \rightarrow H$ with $\psi(x \rho)=x$ for all $x \in H$. Since $H$ is injective, by Result 2.2(ii), $\psi$ extends to an $S$-homomorphism $\tilde{\psi}: S / \rho \rightarrow H$. By Result 2.2(i), $S$ has a left identity element, say, $g$. Put $\tilde{\psi}(g \rho)=h$. It is easily checked that $h S=H$ and $a \rho b(a, b \in S)$ implies $h a=h b$.

Conversely, suppose $S$ satisfies conditions (i) to (iv). By condition (iii), $S,=u S$ for some idempotent $u \in S$. It is clear that $u$ is a left identity element of $S$. Also by condition (i), $S$ has a zero element. So by applying Theorem 1.12 of [10], we see that it suffices to show that for any right congruence $\rho$ on $S$ and for any right ideal $K$ of $S$ such that $k \rho \subseteq K$ for all $k \in K$, each $S$-homomorphism $\psi: K \rho^{\natural} \rightarrow S$ extends to an $S$-homomorphism of $S / \rho$ into $S$. Let $\rho, K, \psi$ be as above. Put $J=\psi\left(K \rho^{\natural}\right)$. Then $J$ is a right ideal of $S$. By Lemma 2.8(i), $J \subseteq K$. Put $\tilde{\rho}=\rho \cup$ $\{(x, y) \in K \times K \mid \psi(x \rho)=\psi(y \rho)\}$. Since $k \rho \subseteq K$ for all $k \in K$, it follows that $\tilde{\rho}$ is a right congruence on $S$. By Lemma 2.8(iii), $\tilde{\rho} \cap(J \times J)=\iota_{J}$. By condition (iii), there is a principal right ideal $I$ of $S$ in which $J$ is strictly large. Then it follows that $\tilde{\rho} \cap(I \times I)=\iota_{I}$. So by condition (iv), there is an idempotent $h \in S$ such that

$$
h S=I \quad \text { and } \quad a \tilde{\rho} b(a, b \in S) \text { implies } h a=h b .
$$

Consider the set $\left\{\psi(e \rho) \mid e^{2}=e \in J\right\}$ and denote it by $M$. Take any $m, n \in M$. Then $\psi(e \rho)=m, \psi(f \rho)=n$ for some idempotents $e, f \in J$. Then $m n^{-1}=$ $\psi(e \rho)(\psi(f \rho))^{-1}=\psi(e \rho) e(\psi(f \rho) f)^{-1} \sigma \psi(e \rho)(e f)(\psi(f \rho))^{-1}$ (since $S / \sigma$ is a semilattice of groups, by Lemma 2.8(iii)) $\sigma \psi(e f \rho)((\psi(f \rho)) e f)^{-1}=\psi(e f \rho)(\psi(f e f \rho))^{-1}=$ $\psi(e f \rho)(\psi(e f \rho))^{-1}$ (since $E(S)$ is right regular, by Result 2.1(i)) $\in E(S)$. Whence, $m \sigma(n \sigma)^{-1} \in E(S / \sigma)$ for all $m, n \in M$. Thus, $M \sigma^{\natural}$ is a compatible subset of $S / \sigma$. By condition (ii), there is an element $w \in S$ such that $w m^{-1} m \sigma m$ for all $m \in M$. 
Since by Result 2.1(vi), $w m^{-1} \in E(S), w m^{-1} \Re m m^{-1}$, we have

$$
m=w m^{-1} m \text { for all } m \in M \text {. }
$$

Let $e=e^{2} \in J$. By Lemma 2.8(ii), $e^{\Re} \psi(e \rho)$. Then $(\psi(e \rho))^{-1}(\psi(e \rho)) \Re e$, and hence, $(\psi(e \rho))^{-1} \psi(e \rho)=(\psi(e \rho))^{-1} \psi(e \rho) e=e$. So by $(2.4)$, we obtain that we $=$ $\psi(e \rho)$. Consequently, we have

$$
w h a=w a=\psi(a \rho) \text { for all } a \in J .
$$

Now define an $S$-homomorphism $\delta: S \rightarrow S$ by $\delta(s)=w h s(s \in S)$. From (2.3), $\delta$ induces the $S$-homomorphism $\tilde{\delta}: S / \rho \rightarrow S$ such that $\tilde{\delta}(x \rho)=\delta(x)$ for all $x \in S$. Let $k \in K$. Then $\psi(k \rho) \in J$. So it follows from Lemma 2.8(ii) that $\psi(k \rho)=\psi(v \rho)$ for some $v \in J$. Then $k \tilde{\rho} v$. By (2.3), we have $h k=h v$. Hence, $\psi(k \rho)=\psi(v \rho)=$ $w h v($ by $(2.5))=w h k=\tilde{\delta}(k \rho)$. Therefore, $\tilde{\delta}$ is an extension of $\psi$. The proof of the theorem is complete.

From Corollary 2.3 and the proof of Theorem 2.9, we have

COROLlaRY 2.10. Let $E$ be a right regular band. Then $E$ is right self-injective if and only if $E$ satisfies the following conditions (i), (ii) and (iii):

(i) E has a zero element;

(ii) for each right ideal $K$ of $E$, there is a principal right ideal $K^{\prime}$ of $E$ in which $K$ is strictly large;

(iii) for any principal right ideal $I$ and for any right congruence $\rho$ on $E$ with $\rho \cap(I \times I)=\iota_{I}$, there is an element $h \in I$ such that $h E=I$ and $a \rho b(a, b \in E)$ implies $h a=h b$.

LEMMA 2.11. Let $S$ be a right inverse semigroup which is a band of groups. Then

(i) $\mathcal{H}$ is a congruence on $S$,

(ii) $x \in S, e=x x^{-1}$ and $e \geqslant f$ (that is, $e f=f e=f$ ) imply $x f=f x$,

(iii) $x^{-1} h x=h x^{-1} x$ for all $x \in S$ and $h \in E(S)$.

Proof. (i) Obvious.

(ii) Since $x \mathcal{H} e$ and $\mathcal{H}$ is a congruence, it follows that $x f \mathfrak{H} f \mathcal{H} f x$. Then $x f=f(x f)=(f x) f=f x$.

(iii) Let $x \in S, h \in E(S)$. Then $x^{-1} h x=x^{-1}\left(x^{-1} x h x^{-1} x\right) x=\left(x^{-1} x h x^{-1} x\right) x^{-1} x$ (by (ii)) $=x^{-1} x h x^{-1} x=h x^{-1} x$ (by Result 2.1(i)). The lemma is proved.

Now we shall prove a main theorem.

THEOREM 2.12. Let $S$ be a right inverse semigroup which is a band of groups. Then $S$ is right self-injective if and only if both $S / \sigma$ and $E(S)$ are right self-injective. 
Proof. First, suppose $S$ is right self-injective. By Theorem 2.6, $S / \sigma$ is a self-injective inverse semigroup. To see that $E(S)$ is right self-injective, it suffices to prove that $E(S)$ satisfies condition (iii) of Corollary 2.10, by Corollary 2.3. Let $I$ be any principal right ideal of $E(S)$ and let $\rho$ be any right congruence on $E(S)$ with $\rho \cap(I \times I)=\iota_{I}$. Then $I=h E(S)$ for some $h \in E(S)$. Define a relation $\xi$ on $S$ by $a \xi b(a, b \in S)$ if and only if $a^{-1} a \rho b^{-1} b$ and $a h=b h$. Then it is clear that $\xi$ is an equivalence relation on $S$. Let $a, b, x \in S$ with $a \xi b$. Then $a x h=$ $a\left(x x^{-1} x\right) h=a x\left(h x^{-1} x h\right)$ (since $E(S)$ is right regular, by Result 2.1(i)) = $a\left(h x x^{-1}\right) x h$ (by Lemma 2.11(iii)) $=a h x h$. Similarly, $b x h=b h x h$. Since $a h=b h$, we have $a x h=b x h$. On the other hand, $(a x)^{-1} a x=x^{-1} a^{-1} a x$ (by Result 2.1(iii),(iv)) $=a^{-1} a x^{-1} x$ (by Lemma 2.11(iii)). Similarly, $(b x)^{-1} b x=b^{-1} b x^{-1} x$. Since $a^{-1} a \rho b^{-1} b$, we have $(a x)^{-1} a x \rho(b x)^{-1} b x$. Hence, $a x \xi b x$, whence $\xi$ is a right congruence on $S$. Since $a^{-1} a=h a^{-1} a \in I, a=a h a^{-1} a$ for all $a \in h S$ and $\rho \cap(I$ $\times I)=\iota_{I}$, it follows that $\xi \cap(h S \times h S)=\iota_{h S}$. So by Theorem 2.9, there is an idempotent $f \in S$ such that $f S=h S$ and $a \xi b(a, b \in S)$ implies $f a=f b$. Let $x, y \in E(S)$ with $x \rho y$. Then $x h \rho y h$. Since $x h=h x h, y h=h y h$, by Result 2.1(i), we have $(x h, y h) \in \rho \cap(I \times I)$. Hence, $x h=y h$, and hence, $x \xi y$. By the property of $f$, we get $f x=f y$. Also, $f E(S)=h E(S)=I$.

Conversely, suppose that $S / \sigma$ and $E(S)$ are right self-injective. We shall show that $S$ satisfies conditions (i) to (iv) of Theorem 2.9. Condition (i) of Theorem 2.9 follows from Corollary 2.10(i). Condition (ii) of Theorem 2.9 follows from Result 1.1. Now we shall see that $S$ satisfies condition (iii) of Theorem 2.9. Let $K$ be any right ideal of $S$. Then by Corollary 2.10(ii), there is an idempotent $g \in S$ such that $E(S) \cap K$ is strictly large in $g E(S)$. While from Result 2.2(iii) and Result 2.1(vi), there is an idempotent $m \in S$ such that $K \sigma^{\natural}$ is strictly large in $(m S) \sigma^{\natural}$. Now that $(m k) \boldsymbol{\sigma}=k \boldsymbol{\sigma}$ for all $k \in K \cap E(S)$, we have $m k=k$ for all $k \in K \cap$ $E(S)$, by Result 2.1(vi). Hence, $m E(S) \supseteq K \cap E(S)$. Thus, $E(S) \cap K$ is strictly large in $m E(S) \cap g E(S)$. By Result 2.1(ii), $m E(S) \cap g E(S)$ is a principal right ideal of $E(S)$. Hence, $m E(S) \cap g E(S)=g E(S)$, by Result 2.2(iii). This implies $(m S) \sigma^{\natural} \supseteq(g S) \sigma^{\natural}$. So it follows that $K \sigma^{\natural}$ is strictly large in $(g S) \sigma^{\natural}$. Let $a, b \in g S$ such that $a \neq b$, and either $a \notin K$ or $b \notin K$.

Case 1: $a \sigma=b \sigma$. Then by Result 2.1(vii), it must be that $a^{-1} a \neq b^{-1} b$. Since $a^{-1} a, b^{-1} b \in g E(S)$, there is an idempotent $t \in E(S)$ with $a^{-1} a t, b^{-1} b t \in K, a^{-1} a t$ $\neq b^{-1} b t$. From Result 2.1(iii), (iv), we have $a t \neq b t, a t, b t \in K$.

Case 2: $a \sigma \neq b \sigma$. First note that either $a \sigma \notin K \sigma^{\natural}$ or $b \sigma \notin K \sigma^{\natural}$, from Result

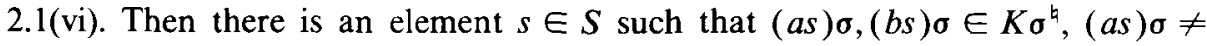
$(b s) \sigma$. Also from Result 2.1(vi), we have $a s, b s \in K$, but $a s \neq b s$. This implies that $K$ is strictly large in $g S$, that is, $S$ satisfies condition (iii) of Theorem 2.9. Finally we shall show that $S$ satisfies condition (iv) of Theorem 2.9. Let $I$ be any right ideal of $S$ and let $\rho$ be any right congruence on $S$ with $\rho \cap(I \times I)=\iota_{I}$. 
Define a relation $\mu$ on $E(S)$ as follows: For any $e, f \in E(S), e \mu f$ if and only if there are elements $x_{1}, x_{2}, \ldots, x_{2 n} \in S$ satisfying

$$
\begin{gathered}
e=x_{1}^{-1} x_{1}, \quad f=x_{2 n}^{-1} x_{2 n}, \\
x_{2 i}^{-1} x_{2 i}=x_{2 i+1}^{-1} x_{2 i+1} \quad(1 \leqslant i \leqslant n-1), \quad x_{2 i+1} \rho x_{2(i+1)} \quad(0 \leqslant i \leqslant n-1) .
\end{gathered}
$$

By using Result 2.1(iii),(iv), we can see easily that $\mu$ is a right congruence on $E(S)$. Let $e, f \in I \cap E(S)$ with $e \mu f$. Then there are elements $x_{1}, x_{2}, \ldots, x_{2 n} \in S$ satisfying (2.6). Then $x_{1} \in I$. Since $x_{1} e \rho x_{2} e$, and $S e \subseteq I$, by Lemma 2.7(ii), we have $x_{1}=x_{1} e=x_{2} e$. Hence, $x_{1} x_{2}^{-1}=\left(x_{2} e\right) x_{2}^{-1}=e\left(x_{2} x_{2}^{-1}\right)$ (by Lemma 2.11(iii)). While, $x_{1} \rho x_{2}$ implies $x_{1} x_{2} x_{2}^{-1} \rho x_{2} \rho x_{1}$. Since $x_{1} \in I$, we have $x_{1} x_{2} x_{2}^{-1}=x_{1}$. Consequently, $x_{1} x_{2}^{-1}=e x_{2} x_{2}^{-1}=\left(x_{1}^{-1} x_{1}\right)\left(x_{2} x_{2}^{-1}\right)=x_{1}^{-1} x_{1}=e$. Put $x=e x_{3}$. Then $x=$ $e x_{3}=\left(x_{1} x_{2}^{-1}\right) x_{3} \rho x_{2}\left(x_{2}^{-1} x_{3}\right)=x_{3} x_{3}^{-1} x_{3}=x_{3} \rho x_{4}$, and $x^{-1} x=x_{3}^{-1} e x_{3}$ (by Result 2.1(iii),(iv)) $=e x_{3}^{-1} x_{3}$ (by Lemma 2.11(iii)) $=e x_{2}^{-1} x_{2}=\left(x_{1}^{-1} x_{1}\right) x_{2}^{-1} x_{2}=x_{1}^{-1} x_{1}$ (as shown above) $=e$. Thus we have $e=x^{-1} x, f=x_{2 n}^{-1} x_{2 n}, x \rho x_{4}, x_{2 i}^{-1} x_{2 i}=$ $x_{2 i+1}^{-1} x_{2 i+1}, x_{2 i+1} \rho x_{2(i+1)}(2 \leqslant i \leqslant n-1)$. By repeating the same argument as above, we can find an element $y \in S$ such that $e=y^{-1} y, y \rho x_{2 n}$ (and $f=x_{2 n}^{-1} x_{2 n}$ ). Then $y, x_{2 n} \in I$. Hence, $y=x_{2 n}$ and hence, $e=f$. This implies that $\mu \cap((E(S)$ $\cap I) \times(E(S) \cap I))=\iota_{E(S) \cap I}$. By Corollary 2.10(iii), there is an element $h \in$ $E(S)$ such that $h E(S)=E(S) \cap I$ and $e \mu f(e, f \in E(S))$ implies $h e=h f$. Then $h S=I$. And let $a, b \in S$ with $a \rho b$. Then $a^{-1} a \mu b^{-1} b$. Hence, $h a^{-1} a=h b^{-1} b$. While, $a h \rho b h$, and $a h, b h \in h S$, by Lemma 2.7(ii). Since $h S=I$, we have $a h=b h$. Hence, $h a=h\left(a a^{-1} a\right)=a h a^{-1} a$ (by Lemma $2.11($ iii) $)=b h b^{-1} b=h b b^{-1} b$ (also by Lemma 2.11(iii)) $=h b$. Therefore, $h a=h b$ for all $a, b \in S$ with $a \rho b$. The proof of the theorem is complete.

Combining Theorem 2.12 with Theorem 7 of [12], Theorem 1.5 of [10] and Corollary 2.3 , we get

THEOREM 2.13. A semigroup is right self-injective and a band of groups if and only if it is isomorphic to the spined product of a right self-injective band and a self-injective semilattice of groups.

EXAMPLE 1. Let $S=\{0, e, f, g, 1\}$ be the semigroup with the following multiplication table:

\begin{tabular}{c|lllll}
$S$ & 0 & $e$ & $f$ & $g$ & 1 \\
\hline 0 & 0 & 0 & 0 & 0 & 0 \\
$e$ & 0 & $e$ & $f$ & $f$ & $e$ \\
$f$ & 0 & $e$ & $f$ & $e$ & $f$ \\
$g$ & 0 & $e$ & $f$ & 1 & $g$ \\
1 & 0 & $e$ & $f$ & $g$ & 1
\end{tabular}


Then $S$ is a right inverse semigroup and a union of groups. Also, $\mathcal{H}$ is not a congruence but a right congruence (that is, $S$ is not $H$-compatible but right $H$-compatible in the sense of [10]). From Theorem 4.1 of [10], both $S / \sigma$ and $E(S)$ are completely right injective, and of course, right self-injective. Now suppose that $S$ is right self-injective. The $S$-homomorphism $\phi:\{0 \mathcal{H}, e \mathcal{H}, f \mathcal{H}\} \rightarrow e S$ with $\phi(e \mathcal{K})=e, \phi(f \mathcal{K})=f$ extends to an $S$-homomorphism $\tilde{\phi}: S / \mathcal{H} \rightarrow e S$. Then it must be that either $e g=e l$ or $f g=f 1$, which is impossible. Therefore, $S$ is not right self-injective.

Example 2. Let $\mathbf{N}$ be the set of all natural numbers and let $A=\cup_{i \in \mathbf{N}} A_{i}$, $A_{i}=\left\{e_{i}, f_{i}\right\}$. Define multiplication on $A$ as follows: For $a \in A_{i}, b \in A_{j}$

$$
a b= \begin{cases}b & \text { if } i \geqslant j, \\ a & \text { if } i>j .\end{cases}
$$

Then $A$ becomes a right regular band which is almost commutative in the sense of [3].

Let $B=\{(m, n) \mid m, n \in \mathbf{N}\}$ be a bicyclic semigroup with multiplication; $(m, n)(k, h)=(m-n+\max (n, k), h-k+\max (n, k))$. Let $A^{0}, B^{0}$ denote the semigroups $A, B$ with zero adjoined. By using Theorem 4.21 of [1], one can construct an ideal extension of $A^{0}$ by $B^{0}$ with the following data:

Let $1_{A^{0}}$ be the identity mapping on $A^{0}$ and let $\rho$ be the right translation of $A^{0}$ such that $0 \rho=0, e_{1} \rho=f_{1}, f_{1} \rho=e_{1}$ and $a \rho=a$ if $a \in A \backslash A_{1}$.

Let $\alpha[\beta]$ be a mapping of $B$ into the semigroup of left [right] translations of $A^{0}$ such that $b \alpha=\lambda_{b}\left[b_{\beta}=\rho_{h}\right]$ for each $b \in B$, where

$$
\lambda_{b}=1_{A^{0}} \quad \text { and } \quad \rho_{b}= \begin{cases}1_{A^{0}} & \text { if } 2 \mid m-n, b=(m, n), \\ \rho & \text { if } 2 \nmid m-n, b=(m, n) .\end{cases}
$$

Let $S$ denote the ideal extension of $A^{0}$ by $B^{0}$ constructed in this way. Then $S$ is a right inverse semigroup which is $H$-compatible in the sense of [10]. Also from Theorem 4.1 of [10], both $S / \sigma$ and $E(S)$ are completely right self-injective, and of course, right self-injective. But it will be seen that $S$ is not right self-injective. Take the right congruence on $S, \mu=(B \times B) \cup \iota_{A^{0}}$ and the right ideal of $S, A^{0}$. Then there is an $S$-homomorphism $\phi: A^{0} \mu^{\natural} \rightarrow A^{0}$ with $\phi(a \mu)=a$ for all $a \in A^{0}$. Suppose that $S$ is right self-injective. Then $A^{0}$ is an injective right ideal of $S$, by Result 2.2(ii). So $\phi$ extends to an $S$-homomorphism $\tilde{\phi}: S / \sigma \rightarrow A^{0}$. Put $\tilde{\phi}((1,1) \mu)$ $=y \in A^{0}$. Then it must be that either $y$ equals $e_{1}$ or $f_{1}$. Since $(1,1) \mu(1,2)$, we have either $e_{1}(1,1)=e_{1}(1,2)$ or $f_{1}(1,1)=f_{1}(1,2)$. In either case, $e_{1}=f_{1}$, a contradiction. Therefore, $S$ is not right self-injective. 


\section{Right self-injective bands}

By Corollary 1.11 of [10], a right self-injective band is right regular. So Corollary 2.10 gives a necessary and sufficient condition for a band to be right self-injective in terms of right ideals and right congruences. We shall present right self-injective bands of two types which are characterized as explicitly as right self-injective normal bands in [10].

According to Hall [3], a band $E$ is said to be almost commutative if for any $e, f \in E$, either $e^{\mathscr{D} f}$ or $f e=e f$. Thus for a right regular band, we have,

LEMMA 3.1. A right regular band $E$ is almost commutative if and only if for any $e, f \in E$, either $e^{\mathscr{R}} f$ or $f e=e f$.

THEOREM 3.2. Let $E$ be an almost commutative band. Then $E$ is right self-injective if and only if $E$ satisfies the following conditions:

(i) $E$ is a right regular band with zero;

(ii) for each right ideal $K$ of $E$, there is a principal right ideal $K^{\prime}$ of $E$ in which $K$ is strictly large.

Proof. The "only if" statement follows from Corollary 1.11 of [10].

To prove the "if" statement it suffices to show that $E$ satisfies condition (iii) of Corollary 2.10. Let $I$ be a principal right ideal of $E$ and let $\rho$ be a right congruence on $E$ with $\rho \cap(I \times I)=\iota_{I}$. Then $I=f E$ for some $f \in E$. If there are no elements $x, y \in E$ such that $x \rho y, x \Re f$ and $(f, y) \notin \Re$, then by Lemma 3.1, either $f a=a f, f b=b f$, or $f a=a, f b=b$ for all $a, b \in E$ with $a \rho b$. Hence, $f a=f b$ because $\rho \cap(I \times I)=\imath$. So we assume that there are elements $x, y \in E$ such that $x \rho y, x \Re f,(f, y) \notin \Re$. Let $a, b \in E$ with $a \rho b$.

Case 1: $(a, x) \notin \mathcal{R},(b, x) \notin \Re$. Then $a x=x a, b x=x b$, by Lemma 3.1. Since $a x \rho b x$, we have $x a=x b$.

Case 2: $(a, x) \in \mathcal{R},(b, x) \notin \Re$. Then xapya. So, $a=x a=y a=a y$ (by Lemma 3.1 and the fact that $(a, y) \notin \mathfrak{R})$. Also, $a x \rho b x$. Hence, $x=a x=b x=x b$. Since $x=x b \rho y b$ and $a=a y \rho b y$, it follows from Lemma 3.1 that either $x \rho y b=$ $b \rho a$ or $x \rho y b=b y \rho a$. In either case, $x \rho a$. Hence, $x=a$. Therefore, $x a=x=x b$ (as shown above). Thus, in any case, we get $x a=x b$. Since $x E=f E=I, E$ satisfies condition (iii) of Corollary 2.10. The proof of the theorem is complete.

From Theorem 3.2 and Corollary 2.3, we have

COROLlaRY 3.3. Let $E$ be a band of idempotents of a right inverse semigroup $S$. If $S$ is right self-injective and $E$ is almost commutative, then $E$ is right self-injective. 
Let $S$ be a semilattice $\Lambda$ of semigroups $S_{\alpha}(\alpha \in \Lambda)$. If $\Lambda$ is a chain, then we shall say that $S$ is a chain of semigroups. In this case, $S_{\alpha} S_{\beta} \subseteq S_{\alpha}$ or $S_{\alpha} S_{\beta} \subseteq S_{\beta}$ for any $\alpha, \beta \in \Lambda$.

THEOREM 3.4. Let $E$ be a chain of right zero semigroups. If $E$ is right self-injective, then $E$ satisfies the following conditions.

(i) E has a zero element.

(ii) For each right ideal $K$ of $E$, there is a principal right ideal $K^{\prime}$ of $E$ in which $K$ is strictly large.

(iii) Each principal right ideal $I$ of $E$ is generated by an element $f \in E$ satisfying $f x=x f$ for all $x \in E \backslash I$.

Proof. Conditions (i), (ii) follows from Result 2.2(i), (iii), respectively. Let $J$ be a principal right ideal of $E$. Then by Result 2.2(ii), $J$ is injective. Define a relation $\xi$ on $E$ by $x \xi y(x, y \in E)$ if and only if either $x, y \in J, x=y$, or $x, y \in E \backslash J$. Then $\xi$ is a right congruence on $E$, since $E$ is a chain of right zero semigroups. Also, $\xi \cap(J \times J)=\iota_{J}$. So we get an $S$-homomorphism $\phi: E / \xi \rightarrow J$ such that $\phi(x \xi)=x$ for all $x \in J$. By Result 2.2(i), $E$ has a left identity element, say, $h$. Put $\phi(h \xi)=a \in J$. Then $a E=J$. Let $t \in E \backslash J$. Then $a t=\phi(h \xi) t=\phi(t \xi)=\phi(h \xi)$ $=a$. Hence, $t a=a=a t$. Thus, $E$ satisfies condition (iii), proving the theorem.

As is shown in Example 3 below, the converse of Theorem 3.4 is not true. In a special case, we have

THEOREM 3.5. Let $E$ be a chain of finite right zero semigroups. Then $E$ is right self-injective if and only if $E$ satisfies conditions (i), (ii) and (iii) of Theorem 3.4.

Proof. The "only if" statement follows from Theorem 3.4.

Conversely, assume that $E$ satisfies conditions (i) to (iii). Since $E$ is a chain of right zero semigroups, it is clear that $E$ is a right regular band. So it suffices to show that $E$ satisfies condition (iii) of Corollary 2.10. Let $I$ be any principal right ideal of $E$ and let $\rho$ be any right congruence on $E$ with $\rho \cap(I \times I)=\iota_{I}$. Then $I=h E$ for some $h \in E$. Put $K=\{x \in E \mid x \rho \cap I \neq \varnothing\}$. Clearly, $K$ is a right ideal of $E$ containing $I$. Now we shall show that for each $x \in K \backslash I$, there is an element $u \in E$ such that $x \rho u, u \Re h$. Let $x \in K \backslash I$. Then $x \rho u$ for some $u \in I$. Then $x h \rho u h$. Since $E$ is right regular, we have $x h, u h \in I$. Hence, $x h=u h$. Also, since $E$ is a chain of right zero semigroups, we have $x h \Re h, u h \Re u$. Hence, $h \Re u$. Thus, $x \rho u$ and $u \Re h$.

Next we shall show that there is an element $w \in E$ such that $w \Re h$ and $w k \rho k$ for all $k \in K \backslash I$. First note that for any $a, b \in E$, either $a \in b E$ or $b \in a E$. Let 
$a, b \in K \backslash I$. By the above, $a \rho u, b \rho v$ for some $u, v \in E$ with $u \Re v$ q $h$. If $a \in b E$, then $a=b a \rho v a$. Thus, $a \rho v a$ and $b \rho v b$. Also, if $b \in a E$, then $a \rho u a$ and $b \rho u b$. So since the $R$-class containing $h$ is a finite set, we can find an element $w \in E$ such that $w \Re h$ and $w k \rho k$ for all $k \in K \backslash I$. Since $\rho \cap(I \times I)=\iota_{I}$, it follows that

$$
w k=w p \quad \text { for all } k, p \in K \text { with } k \rho p .
$$

By conditions (ii), (iii), there is an element $f \in E$ such that $f x=x f$ for all $x \in E \backslash f E$ and $K$ is strictly large in $f E$. Then it must be that $f E=\{f\} \cup K$. Suppose that $m \in f E \backslash K$ with $m \neq f$. Since $K$ is strictly large in $f E, m s, f s \in K$, $m s \neq f s$. Since $E$ is a chain of right zero semigroups, we have $s \in K$ and $m s=f s=s$. This is a contradiction. Thus, $f E=\{f\} \cup K$. Put $w f=z$. Let $c, d \in E$ with $c \rho d$.

Case 1: $f E=K$. From the definition of $K$, we get either $c, d \in K$ or $c, d \in E \backslash K$. In the former case, clearly, $z c=z d$. In the latter case, $f c=c f \rho d f=f d$ and $f c, f d \in K$. By (3.1), $w f c=w f d$, that is, $z c=z d$. In either case, we have $z c=z d$.

Case 2: $f \notin K$. As shown above, we have either $c, d \in K$ or $c, d \in E \backslash K$. In the former case, clearly, $z c=z d$. In the latter case, we get $f c \Re f d \Re f$. Since $f E=K \cup$ $\{f\}$, it follows that $f=f c=f d$. Hence, $z c=z d$.

In either case, we have $z c=z d$. Therefore, $z c=z d$ for all $c, d \in E$ with $c \rho d$. On the other hand, $w \Re h$ and $w \in f E$. Since $E$ is a chain of right zero semigroups, it follows that $h \Re w \Re w f=z$. Hence, $z E=h E=I$. Thus, $E$ satisfies condition (iii) of Corollary 2.10. The proof of the theorem is complete.

ExAmple 3. Let $B_{0}=\{0\}, B_{1}=\left\{b_{1}, b_{2}, b_{3}, \ldots\right\}, B_{2}=\left\{a_{2}\right\}, B_{3}=\left\{a_{3}\right\}, \ldots$, and $B_{\infty}=\{1\}$. Let $B$ be a disjoint union of $B_{i}(i \geqslant 0)$ and $B_{x}$. Define multiplication on $B$ as follows:

$$
\begin{gathered}
0 x=x 0=0, \quad 1 x=x 1=x \text { for all } x \in B, \\
x b_{i}=b_{i} \text { for all } x \in B \backslash B_{0} \text { and for all } i \geqslant 1, \\
b_{i} a_{j}=b_{\min (i, j)} \quad \text { for all } i \geqslant 1 \text { and } j \geqslant 2, \\
a_{j} a_{k}=a_{\min (j, k)} \quad \text { for all } j, k \geqslant 2 .
\end{gathered}
$$

Then $B$ becomes a chain of right zero semigroups satisfying conditions (i) to (iii) of Theorem 3.4. But it will be seen that $B$ is not right self-injective. Take the right congruence on $B, \rho=\left\{\left(a_{i}, b_{i}\right),\left(b_{i}, a_{i}\right) \mid i \geqslant 2\right\} \cup \iota_{B}$ and the right ideal of $B$, $B_{0} \cup B_{1}$. Then there is a $B$-homomorphism $\phi:\left(B_{0} \cup B_{1}\right) \rho^{\natural} \rightarrow B_{0} \cup B_{1}$ with $\phi(x \rho)=x$ for all $x \in B_{0} \cup B_{1}$. Suppose that $B$ is right self-injective. By Result 2.2(ii), $B_{0} \cup B_{1}$ is injective. So, $\phi$ extends to a $B$-homomorphism $\tilde{\phi}: B / \rho \rightarrow B_{0} \cup$ $B_{1}$. Put $\tilde{\phi}(1 \rho)=b_{m}$. Then, $b_{m}=b_{m} a_{m+1}=\tilde{\phi}\left(a_{m+1} \rho\right)=\tilde{\phi}\left(b_{m+1} \rho\right)=b_{m+1}$, a contradiction. Therefore, $B$ is not right self-injective. 
EXAMPLE 4. We give an example of a finite right regular band satisfying conditions (i) to (iii) of Theorem 3.4 and which is not right self-injective.

Let $E$ be the band with the following multiplication table:

\begin{tabular}{c|ccccccccc}
$E$ & $a$ & $b$ & $c$ & $c_{1}$ & $z$ & $c_{2}$ & $d$ & 0 & 1 \\
\hline$a$ & $a$ & 0 & $c_{1}$ & $c_{1}$ & $z$ & 0 & $d$ & 0 & $a$ \\
$b$ & 0 & $b$ & $c_{2}$ & 0 & 0 & $c_{2}$ & $d$ & 0 & $b$ \\
$c$ & $c_{1}$ & $c_{2}$ & $c$ & $c_{1}$ & $z$ & $c_{2}$ & 0 & 0 & $c$ \\
$c_{1}$ & $c_{1}$ & 0 & $c_{1}$ & $c_{1}$ & $z$ & 0 & 0 & 0 & $c_{1}$ \\
$z$ & $z$ & 0 & $c_{1}$ & $c_{1}$ & $z$ & 0 & 0 & 0 & $z$ \\
$c_{2}$ & 0 & $c_{2}$ & $c_{2}$ & 0 & 0 & $c_{2}$ & 0 & 0 & $c_{2}$ \\
$d$ & $d$ & $d$ & 0 & 0 & 0 & 0 & $d$ & 0 & $d$ \\
0 & 0 & 0 & 0 & 0 & 0 & 0 & 0 & 0 & 0 \\
1 & $a$ & $b$ & $c$ & $c_{1}$ & $z$ & $c_{2}$ & $d$ & 0 & 1
\end{tabular}

Then $E$ satisfies conditions (i) to (iii). But it will be seen that $E$ is not right self-injective. Take the right congruence on $E, \rho=\iota_{E} \cup\{(a, z),(z, a),(d, 0)$, $(0, d)\}$. Then there is an $E$-homomorphism $\phi:\left(a E \cup c_{2} E\right) \rho^{\natural} \rightarrow c E$ such that $\phi(a \rho)=z, \phi\left(c_{2} \rho\right)=c_{2}$. Suppose that $E$ is right self-injective. By Result 2.2(ii), $c E$ is injective. So, $\phi$ is extended to an $E$-homomorphism $\tilde{\phi}: E / \rho \rightarrow c E$. Then $\tilde{\phi}(1 \rho)=c$. Hence, $c_{1}=c a=\phi(a \rho)=z$, a contradiction. Therefore, $E$ is not right self-injective.

\section{Congruence and representation extension properties}

For a set $X$, let $\mathscr{T}(X)\left[\sigma^{*}(X)\right]$ denote the full transformation semigroup on $X$ with operation on $X$ on the right [left] and composition from left to right [right to left].

Following Hall [4], a subsemigroup $U$ of a semigroup $S$ is said to have the [anti-] representation extension property in $S$ if for any set $X$ and any representation (that is, homomorphism) $\alpha: U \rightarrow \sigma(X)\left[\widetilde{\sigma}^{*}(X)\right]$, there are a set $Y$ disjoint from $X$ and a representation $\beta: S \rightarrow \mathcal{G}(X \cup Y)\left[\mathcal{F}^{*}(X \cup Y)\right]$ such that $\beta_{u} \mid X=\alpha_{u}$ for all $u \in U$. A semigroup $U$ is said to have the [anti-] representation extension property if for every semigroup $S$ with $U$ as a subsemigroup, $U$ has the [anti-] representation extension property in $S$.

RESUlt 4.1 [4, Theorem 17]. Let $U$ be an subsemigroup of any semigroup $S$. Then $U$ has the representation extension property in $S$ if and only if for every semigroup $T$ containing $U$ as a subsemigroup, there are no distinct elements $t, t^{\prime} \in T$ such that for 
some $s_{1}, \ldots, s_{n-1} \in S, t_{1}, \ldots, t_{n} \in T, u_{1}, v_{1}, \ldots, u_{n}, v_{n} \in U$,

$$
\begin{gathered}
t=t_{1} u_{1}, \quad t_{n} v_{n}=t^{\prime}, \\
u_{1}=v_{1} s_{1}, \quad u_{2} s_{1}=v_{2} s_{2}, \quad u_{3} s_{2}=v_{3} s_{3}, \ldots, u_{n} s_{n-1}=v_{n}, \\
t_{1} v_{1}=t_{2} u_{2}, \quad t_{2} v_{2}=t_{3} u_{2}, \ldots, t_{n-1} v_{n-1}=t_{n} u_{n} .
\end{gathered}
$$

(This set of equations is called a length $n$ zigzag of type II (a) over $U$ from $t \in T$ to $t^{\prime} \in T$ with spline $u_{1}, v_{1}, \ldots, u_{n}, v_{n}$ (in that order).)

THEOREM 4.2. A left [right] self-injective semigroup has the [anti-] representation extension property.

Proof. Let $U$ be a left self-injective semigroup, and $S, T$ semigroups containing $U$ as a subsemigroup. Suppose that there is such a length $n$ zigzag of type II (a) over $U$ from $t \in T$ to $t^{\prime} \in T$ as in (4.1). There is a $U$-homomorphism $\psi$ of the left $U$-system $S$ onto the injective left $U$-system with $\psi \mid U=\iota_{U}$. Hence we get $u_{1}=v_{1}\left(s_{1} \psi\right), u_{2}\left(s_{1} \psi\right)=v_{2}\left(s_{2} \psi\right), u_{3}\left(s_{2} \psi\right)=v_{3}\left(s_{3} \psi\right), \ldots, u_{n}\left(s_{n-1} \psi\right)=v_{n}$. Then it follows from equations (4.1) that $t=t^{\prime}$. The required result follows from Result 4.1.

Following Hall [4], we say that a subsemigroup $U$ of a semigroup $S$ has the right [left] congruence extension property in $S$ if for any right [left] congruence $\rho$ on $U$, there is a right [left] congruence $\xi$ on $S$ with $\xi \cap(U \times U)=\rho$. A semigroup $U$ is said to have the right [left] congruence extension property if for every semigroup $S$ with $U$ as a subsemigroup, $U$ has the right [left] congruence extension property in $S$.

The following result is a consequence of Theorem 4.2 and Theorem 9 of [4]. Here we shall prove it directly.

THEOREM 4.3. A left [right] self-injective semigroup has the right [left] congruence extension property.

Proof. Let $U$ be a left self-injective semigroup and let $S$ be a semigroup containing $U$ as a subsemigroup. Take any right congruence $\rho$ on $U$. Define a relation $\xi$ on $S$ as follows: for any $a, b \in S, a \xi b$ if and only if either $a=b$ or there are elements $x_{1}, x_{2}, \ldots, x_{n} \in S^{l}, u_{1}, \ldots, u_{2 n} \in U$ such that

$$
\begin{array}{cl}
a=u_{1} x_{1}, & u_{2 n} x_{n}=b, \\
u_{2 i} x_{i}=u_{2 i+1} x_{i+1} & (1 \leqslant i \leqslant n-1) \quad \text { and } \\
u_{2 i-1} \rho u_{2 i} & (1 \leqslant i \leqslant n) .
\end{array}
$$


Then $\xi$ is a right congruence on $S$. Since $U$ is left self-injective, there is a $U$-homomorphism $\psi$ of the left $U$-system $S$ onto the left $U$-system $U$ with $\psi \mid U=\iota_{U}$. Let $a, b, x_{i}, u_{i}$ be as in (4.2). Then $a \psi=u_{1}\left(x_{1} \psi\right), u_{2 n}\left(x_{n} \psi\right)=b \psi$, $u_{2 i}\left(x_{i} \psi\right)=u_{2 i+1}\left(x_{i+1} \psi\right) \quad(1 \leqslant i \leqslant n-1)$. Thus if follows that $a \psi \rho b \psi$. So, if $a, b \in U$, then $a \rho b$. This means that $\xi \cap(U \times U) \subseteq \rho$. It is clear that $\xi \cap(U \times$ $U) \supseteq \rho$. Therefore, $\xi \cap(U \times U)=\rho$. The proof of the theorem is complete.

REMARKS. (i) It is known [4] that each semigroup with the representation extension property is absolutely closed, so it follows from Theorem 4.2 that a left [right] self-injective semigroup is absolutely closed. This has been proved also in [9].

(ii) Hall [5] showed that the semigroup $\mathscr{P} \mathcal{T}_{X}$ of all partial transformations of a set $X$ is absolutely closed. This can be also proved by using Theorem 2 of [9] and Theorem 4.4 below.

For any set $X$, consider $X$ as a right zero semigroup (that is, $x y=y$ for all $x, y \in X)$. Take the semigroup $X^{0}$ obtained from $X$ by adjoining a zero 0 and consider the semigroup $\mathcal{P}\left(X^{0}\right)$ of all right translations of $X^{0}$ written on the right. Then $\mathscr{P}\left(X^{0}\right)$ is isomorphic to $\mathscr{P F}_{X}$. Thus, by the left-right dual of Theorem 3 in [8], we have

THEOREM 4.4. The semigroup of all partial transformations of a set (written on the right) is a left self-injective semigroup.

Combining Theorem 4.2 with Theorem 4.4, we have

COROLlaRY 4.5 [5, Remark 1]. The semigroup of all partial transformations of a set (written on the right) has the representation extension property.

\section{References}

[1] A. H. Clifford and G. B. Preston. The algebraic theory of semigroups, Vol. 1 (Math. Surveys, No. 7, Amer. Math. Soc., Providence, R.I., 1961).

[2] T. E. Hall, 'On regular semigroups whose idempotents form a subsemigroup', Bull, Austral. Math. Soc. 1 (1969), 195-208.

[3] T. E. Hall, 'Almost commutative bands', Glasgow Math. J. 13 (1972), 176-178.

[4] T. E. Hall, 'Representation extension and amalgamation for semigroups', Quart. J. Math. Oxford Ser. 29 (1978), 309-334.

[5] T. E. Hall, 'Epimorphisms and dominions', Semigroup Forum 24 (1982), 271 -283.

[6] N. Kimura, 'The structure of idempotent semigroups. (I)', Pacific J. Math. 8 (1958), 257-275.

[7] B. M. Schein, 'Injective monars over inverse semigroups', Algebraic theory of semigroups, pp. 519-544 (Colloquia Math. Soc. Janos Bolyai 20, Szeged (Hungary), 1976). 
[8] K. Shoji, 'Injective hulls of certain right reductive semigroups $S$ as right $S$-systems', Mem. Fac. Sci. Shimane Univ. 14 (1980), 25-34.

[9] K. Shoji, 'Right self-injective semigroups are absolutely closed', Mem. Fac. Sci. Shimane Univ. 14 (1980), 35-39.

[10] K. Shoji, 'On right self-injective regular semigroups', Semigroup Forum 25 (1982), 51-71.

[11] M. Yamada, 'Strictly inversive semigroups', Sci. Rep. Shimane Univ. 13 (1964), 128-138.

[12] M. Yamada, 'On a regular semigroup in which the idempotents form a band', Pacific J. Math. 33 (1970), 26l-272.

\section{Department of Mathematics}

Shimane University

Matsue, Shimane, Japan 